\title{
ROBUST LFT-LPV Hळ CONTROL OF AN UNDERACTUATED INVERTED PENDULUM ON A CART WITH OPTIMAL WEIGHTING FUNCTIONS SELECTION BY GA AND ES
}

\author{
Seif-EI-Islam HASSENI," Latifa ABDOU*
}

\begin{abstract}
*LMSE Laboratory, Electrical Engineering Department, University of Biskra, BP 145 RP, 07000, Biskra, Algeria
${ }^{*}$ LI3CUB Laboratory, Electrical Engineering Department, University of Biskra, BP 145 RP, 07000, Biskra, Algeria
\end{abstract}

seif.hasseni@univ-biskra.dz, l.abdou@univ-biskra.dz

received 23 March 2019, revised 18 January 2021, accepted 21 January

\begin{abstract}
This article investigates the robust stabilization and control of the inverted pendulum on a cart against disturbances, measurement noises, and parametric uncertainties by the LFT-based LPV technique (Linear-Fractional-Transformation based Linear-ParameterVarying). To make the applying of the LPV technique possible, the LPV representation of the inverted pendulum on a cart model is developed. Besides, the underactuated constraint of this vehicle is overcome by considering both degrees of freedom (the rotational one and the translational one) in the structure. Moreover, the selection of the weighting functions that represent the desired performance is solved by two approaches of evolutionary algorithms; Genetic Algorithms (GA) and Evolutionary Strategies (ES) to find the weighting functions' optimal parameters. To validate the proposed approach, simulations are performed and they show the effectiveness of the proposed approach to obtain robust controllers against external signals, as well as the parametric uncertainties.
\end{abstract}

Keywords: Inverted Pendulum on a cart, Robust Control, Underactuated Systems, Linear Parameter Varying Systems, $\mathrm{H}_{\infty}$

\section{INTRODUCTION}

\subsection{Context and Motivations}

The $\mathrm{H}_{\infty}$ control is one of the efficient control approaches on the robustness problem. Its objective is to minimize the gain between the external input signals and the so-called output signals (Zhou and Doyle, 1998). For the first time, it is developed for the Linear Time-Invariant systems (LTI). An extension of the $\mathrm{H}_{\infty}$ theory for the Linear Parameter Varying systems (LPV) has been developed. It has investigated the control synthesis and stability analysis of the LPV systems (Iwasaki and Shibata, 2001; Scherer, 2001; Wu, 2001). There are three kinds of LPV representations. First, the polytopic approach; the parameters are used at each vertex in the polytope. The main disadvantage of this approach is that exponential number of controllers are required, therefore, solving a large number of Linear Matrices Inequalities (LMI) and the computational effort is then expensive (Salhi et al., 2015; Liu, 2017). Second, gridding linearization; with this approach, there are simple controllers to implement, but, because the discretization on the parameters space is not well-defined (Wu et al., 1996), we may get infinity controllers. Third and last one; the LPV system with Linear Fractional Transformation (LFT) is common in separating the uncertainties from the nominal model in robust control. In this class, the same structure (LFT) is used with varying parameters. The main advantage of this approach is that minimal LMls are solved to design a single controller. This controller has a selfscheduled structure around the parameters (Packard, 1994; Apkarian and Gahinet, 1995). The LPV theory shows efficiency in overcoming the complexity caused by the nonlinearity, especially the LFT representation, which we used in this paper. Moreover, researches were carried out about LPV systems in robust control because it's rigorous in robust stability. So, rather than divide the nonlinear system in a set of LTI systems according to their operating points, the LPV system is developed by considering the nonlinearities as varying parameters, it is called here a quasi-LPV system (Shamma and Athans, 1991; Abbas et al., 2014).

On the other hand, the presented paper aims to investigate the design of a robust LPV controller for an inverted pendulum on a cart. The inverted pendulum is a classical benchmark, which can be considered as the simplest robotic system with one rigid body and one joint (Boubaker, 2013). The inverted pendulum could be considered as an ideal nonlinear system with a stable equilibrium point when the pendulum is in the pending position and an unstable equilibrium point when the pendulum is in the upright position. When the system is moved up from the pending position to the upright position, the model is strongly nonlinear with the pendulum angle. Besides, with two Degrees Of Freedom (DOF); and only the horizontal force, the inverted pendulum is the simplest Underactuated Mechanical System (UMS). Therefore, the inverted pendulum seems to be the platform for the implementation of different nonlinear and linear approaches; augmented PID controller (Siradjuddin et al., 2018), fuzzy logic control (El-Bardini and ElNagar, 2014), sliding mode (Park and Chwa, 2009), predictive control (Ohhira and Shimada, 2017), and optimal control (Prasad et al., 2014).

\subsection{Related Works}

Many recent papers have interested the LPV techniques. For instance, in Tasoujian et al. (2020), the authors considered a parameter-varying model with delays to describe the dynamics of mean arterial blood pressure. Next, they designed a gainscheduled output feedback LPV controller against disturbances and norm-bounded uncertainties to develop and regulate a realtime mean arterial blood pressure response of patients via time 
delay LPV control technique. The LPV techniques are commonly used in sensible systems that need rigorous rejection of disturbances, uncertainties' effects, and faults like unmanned aerial vehicles (López-Estrada et al., 2016; Hasseni and Abdou, 2018). The authors of (Nguyen et al., 2020) constructed a conceptual model of narrow tilting vehicles in a polytopic LPV form, and they then proposed a static output feedback control method as the simplest structure to avoid the use of costly vehicle sensors. In (Xu et al., 2019), a novel robust fault detection approach is proposed. Besides, an unknown input observer is designed for LPV systems with both state and output scheduled by inexact scheduling variables. Here, the stability conditions for the proposed approaches are established via LMI. In Rotondo et al. (2018), a discrete-time LPV unknown input observer is proposed for the diagnosis of actuator faults and ice accretion in unmanned aerial vehicles. The effectiveness of the proposed approach is validated by simulations illustrating the diagnosis of actuator faults and icing in a small unmanned aerial vehicle. In Li et al. (2019), the authors designed a novel fault detection filter for a class of continuoustime LPV systems. This filter is transformed into an $\mathrm{H}_{\infty}$ filtering problem for the filtering error system with uncertain parameters. In Liu et al. (2019), an LPV model for the aero-engine dynamics is constructed. Next, a theoretical sufficiency criterion is provided to guarantee $H_{\infty}$ performance based on LMI. The simulations showed the validity of the proposed strategy by reducing the computational cost and avoiding false switching due to disturbances. The authors of (Yang et al., 2020) constructed an LPV model of the proton-exchange-membrane-fuel-cell system to describe its behaviour and to reduce the computation cost. Besides, this LPV model is used to propose an augmented state observer for simultaneously estimating the states and component faults. The robustness is guaranteed against disturbances and measurement noises. In Alcalá et al. (2020), to develop a Model Predictive Controller that can be calculated online with reduced computational cost, the authors used the LPV theory to model and to control an autonomous vehicle. On the other hand, the problem of the best trajectory is solved by an optimal offline trajectory planner.

\subsection{Challenges and Contributions}

In previous work (Hasseni and Abdou, 2017), the translational DOF has been eliminated by taking a reduced model; the model was presented by two states $(\theta, \dot{\theta})$, the objective was to stabilize the pendulum in null angle with robustness against disturbances and uncertainties. In this work, the novelty is presented within these three points; include the friction effect and consider the velocity as a state (a), getting optimal weighting functions (b) and both of the two DOF $(\theta$ and $x)$ is stabilized and controlled simultaneously (c).

a) Unlike Hasseni and Abdou (2017), in this work, we manipulate with considering the friction effect, so, the translational velocity has been included as a state. We note that we don't consider the translational position $(x)$ as a state because it isn't necessary to measure or estimate the position of the pendulum's cart (i.e., Segway), where the objective is to stabilize it because the rider can move it by inclining himself.

b) The technique of the controller design (LFT-based LPV $H_{\infty}$ ) is applied here. One of the problems we face on the design is the weighting functions' selection, which depends on experimental skills. Many researchers have been interested in this issue (Zhou and Doyle, 1998; Beaven et al., 1996; Hu et al., 2000), but there is no general methodology to select the weighting functions. This problem appears on complex systems when the performance specification is not (or is difficult to) defined. When we impose such optimal desired performance presented by the weighting functions, the $\mathrm{H}_{\infty}$ controller design process maybe failed to find a suitable controller for such performance, the solution is that we have to demean the performance specification. Nature-inspired stochastic optimization tools have been used in many works to get the weighting functions' optimal parameters with the existing $\mathrm{H}_{\infty}$ controller, for the LTI system (Alfaro-Cid et al., 2008), for a polytopic-LPV system (Do et al., 2011; Vu et al., 2017), and for an LFT-LPV system (Hasseni and Abdou, 2020). These tools are more required in the multi-objective problem, which is very common in the controller designing process. To get the weighting functions' optimal parameters, we use in this paper two evolutionary algorithms; first, the Genetic Algorithm (GA), which is based on Darwinian evolution, and is created by $\mathrm{Hol}$ land (Holland, 1992). Second, the Evolutionary Strategies (ES), the first evolutionary algorithm created by Rechenberg (1973), which is based on biological evolution.

c) With 2DOF ( $x$ and $\theta$ ) and just one input (Force), the inverted pendulum is considered as an Underactuated Mechanical System (UMS). Based on previous works (Hasseni and Abdou, 2017; Choukchou-Braham et al., 2014a), we conclude that the inverted pendulum is a kind of system that could not be transformed to chain structured and be controlled in cascade strategy. Therefore, in this paper, the global stabilization of the vehicle is investigated under this constraint. We design a controller that stabilizes the two DOF $(\theta$ and $x)$ simultaneously.

\subsection{The Paper Outline}

The outline of the paper is as follows: In Section 2, we introduce the proposed vehicle to control, the inverted pendulum on a cart. Besides, we present its model and develop its equivalent LFT-LPV representation. In Section 3, we will show the selected evolutionary algorithms, GA and ES, and the characteristics of such techniques we use. In Section 4, after getting the closedloop system, including the disturbance, the measurement noise, and the weighting functions, we will apply the optimization process to get the weighting functions' optimal parameters considering the angle as the only output. The underactuated degree of freedom is taken into account in Section 5, we will show the ultimate simulation results of stabilizing the system. Finally, our conclusion is presented in Section 6.

\section{THE INVERTED PENDULUM ON A CART}

\subsection{The mathematic model of the inverted pendulum on a cart}

A dynamic model of any mechanical system can be derived from Lagrange-Euler formulation. We can describe the dynamic model of the mechanical system as follows:

$M(q) \ddot{q}+C(q, \dot{q}) \dot{q}+G(q)=u$ 
where $q$ is the degree of freedom coordinate. $\dot{q}$ and $\ddot{q}$ are its first and second derivatives. $M(q)$ is the symmetric definite positive inertia matrix. The term $C(q, \dot{q})$ is the centrifugal and Coriolis matrix. The term $G(q)$ is the gravitational torques vector, and $u$ is the inputs.

The model of the inverted pendulum on a cart is a benchmark for many autonomous vehicles, that is, Segway (Younis and Abdelati, 2009), which is a vehicle having two degrees of freedom; one is relating on the angular coordinate $(\theta)$ and the other is relating on the translational coordinate $(x)$, but the only applied input is the linear thrust force $(F)$. The control objective is to stabilize the pendulum in the null angle whatever the initial angle (Fig. 1).

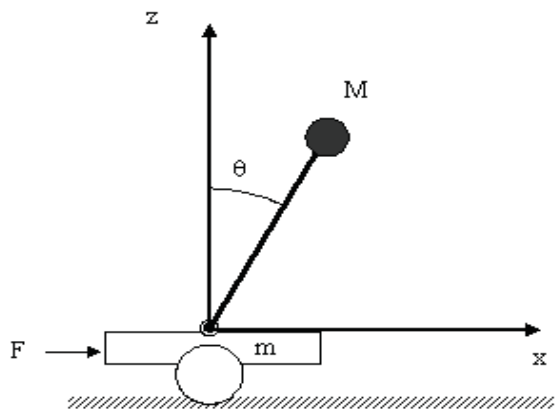

Fig. 1. Geometric scheme of an inverted pendulum on a cart

The dynamic model of the considered system, the inverted pendulum on a cart, is presented in the next expression (Fiacchini et al., 2006; Raffo et al., 2007):

$$
\begin{aligned}
& {\left[\begin{array}{cc}
M+m & M l \cos (\theta) \\
M l \cos (\theta) & M l^{2}
\end{array}\right]\left[\begin{array}{c}
\ddot{x} \\
\ddot{\theta}
\end{array}\right]+\left[\begin{array}{cc}
b & -M l \sin (\theta) \dot{\theta} \\
0 & 0
\end{array}\right]\left[\begin{array}{c}
\dot{x} \\
\dot{\theta}
\end{array}\right]+} \\
& {\left[\begin{array}{c}
0 \\
-M g l \sin \theta
\end{array}\right]=\left[\begin{array}{c}
F \\
0
\end{array}\right]}
\end{aligned}
$$

Where $m$ is the mass of the cart. $M$ is the pendulum mass that presents here the mass of the rider who guides the vehicle, $l$ is the pendulum length, $g$ is the gravity constant, $b$ is the linear friction force coefficient, and $F$ is the linear force applied in the vehicle. The objective of this research is the robust control of this nonlinear system by using the LPV technique. We will create the nonlinear model of the inverted pendulum (2) as an LPV with LFT representation in the next subsection.

\subsection{The LFT-LPV representation of the inverted pendulum on a cart}

As we have mentioned, there are different presentations of an LPV system; polytopic, gridding, and LFT. We use the last one to present the inverted pendulum model because we are getting a single scheduled controller. We have to notice that the controller has the same representation of the system.

We extract the two equations from (2):

$$
\left\{\begin{array}{l}
(M+m) \ddot{x}+M l \cos (\theta) \ddot{\theta}+b \dot{x}-M l \sin (\theta) \dot{\theta}^{2}=F \\
M l \cos (\theta) \ddot{x}+M l^{2} \ddot{\theta}-M g l \sin (\theta)=0
\end{array}\right.
$$

We face the issue that the term $\left(M l \sin (\theta) \dot{\theta}^{2}\right)$ makes the applying of the LFT-LPV technique difficult. To avoid this problem, we change the generated signal by the controller. The new dynamic model is:

$$
\begin{aligned}
& \left\{\begin{array}{l}
(M+m) \ddot{x}+M l \cos (\theta) \ddot{\theta}+b \dot{x}=u \\
M l \cos (\theta) \ddot{x}+M l^{2} \ddot{\theta}-M g l \sin (\theta)=0
\end{array}\right. \\
& \quad \text { In which: } \\
& u=F+M l \sin (\theta) \dot{\theta}^{2}
\end{aligned}
$$

By replacing one equation of (4) with the other, we take the nonlinearities as varying parameters. After some mathematical operations, we extract the LFT-LPV state space (6) and its structure (Fig. 2):

$\left[\begin{array}{c}\dot{x} \\ z_{\rho} \\ y\end{array}\right]=\left[\begin{array}{ccc}\mathcal{A} & \mathcal{B}_{\rho} & \mathcal{B}_{1} \\ \mathcal{C}_{\rho} & \mathcal{D}_{\rho \rho} & \mathcal{D}_{\rho 1} \\ \mathcal{C}_{1} & \mathcal{D}_{1 \rho} & \mathcal{D}_{11}\end{array}\right] \cdot\left[\begin{array}{c}x \\ w_{\rho} \\ u\end{array}\right]$
$w_{\rho}=\Theta z_{\rho}$

where $x$ is the states' vector, $x=\left[\begin{array}{lll}\theta & \dot{\theta} & \dot{x}\end{array}\right]^{T} . u$ is the input. $z_{\rho}$ and $w_{\rho}$ are the inputs and outputs of the parameters block $\Theta$.

The matrices:

$$
\begin{aligned}
\mathcal{A}=\left[\begin{array}{ccc}
0 & 1 & 0 \\
\frac{g}{l} & 0 & \frac{b}{(M+m) l} \\
0 & 0 & \frac{-b}{(M+m)}
\end{array}\right], \mathcal{B}_{\rho} \\
=\left[\begin{array}{cccc}
0 & 0 & 0 & 0 \\
\frac{g}{l} \frac{1}{(M+m) l} & 0 & \frac{M}{(M+m)} \\
0 & 0 & \frac{M l}{(M+m)} & 0
\end{array}\right],
\end{aligned}
$$$$
\mathcal{B}_{1}=\left[\begin{array}{c}
0 \\
\frac{-1}{(M+m)} \\
\frac{1}{(M+m)}
\end{array}\right], \mathcal{C}_{\rho}=\left[\begin{array}{lll}
1 & 0 & 0 \\
0 & 0 & b \\
\frac{g}{l} & 0 & \frac{b}{(M+m) l} \\
0 & 0 & 0
\end{array}\right], \mathcal{C}_{1}=
$$$$
\left[\begin{array}{lll}
1 & 0 & 0
\end{array}\right] \text {, }
$$

$$
\begin{aligned}
& \mathcal{D}_{\rho \rho}=\left[\begin{array}{cccc}
0 & 0 & 0 & 0 \\
0 & 0 & 0 & 0 \\
\frac{g}{l} & \frac{1}{(M+m) l} & 0 & \frac{M}{(M+m)} \\
0 & 0 & 1 & 0
\end{array}\right], \mathcal{D}_{\rho 1}=\left[\begin{array}{c}
0 \\
-1 \\
-1 \\
\frac{(M+m)}{0}
\end{array}\right], \\
& \mathcal{D}_{1 \rho}=\left[\begin{array}{llll}
0 & 0 & 0 & 0
\end{array}\right], \mathcal{D}_{11}=0, \Theta=\left[\begin{array}{cccc}
\rho_{1} & 0 & 0 & 0 \\
0 & \rho_{2} & 0 & 0 \\
0 & 0 & \rho_{3} & 0 \\
0 & 0 & 0 & \rho_{4}
\end{array}\right]
\end{aligned}
$$

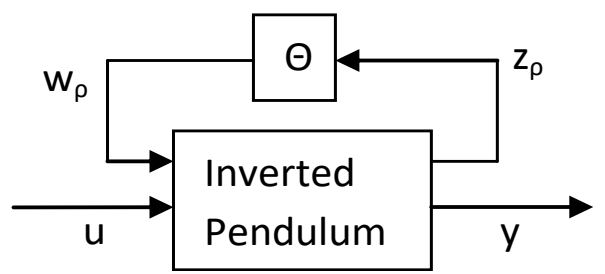

Fig. 2. LFT-LPV scheme of the inverted pendulum

Before designing a controller to stabilize such an LPV system, we need to provide the limit bound of each parameter. All the parameters; $\rho_{1} \ldots \rho_{4}$ are dependent on $\theta$. 
Theoretically, $-90^{\circ} \leq \theta \leq 90^{\circ}$ but to avoid the noncontrollability, we have to reduce the bound to $-80^{\circ} \leq \theta \leq$ $80^{\circ}$. Table 1 presents the range of each parameter.

Tab. 1. The ranges of the varying parameters

\begin{tabular}{|l|c|l|l|}
\hline Parameter & Description & Min. value & Max. value \\
\hline $\boldsymbol{\rho}_{1}$ & $\left(\frac{\sin \theta}{\theta}\right)-1$ & -0.3 & 0 \\
\hline $\boldsymbol{\rho}_{2}$ & $\cos \theta-1$ & -0.83 & 0 \\
\hline $\boldsymbol{\rho}_{3}$ & $\cos \theta$ & 0.17 & 1 \\
\hline $\boldsymbol{\rho}_{4}$ & $\cos ^{2} \theta$ & 0.03 & 1 \\
\hline
\end{tabular}

We notice that in (6)-(7), we didn't generate the closed-loop system yet, just convert the plant (4) to LFT-LPV representation. We need to interconnect the plant with the disturbances and the weighting functions. Our contribution is to select the weighting functions parameters by the nature-inspired algorithms (GA and $E S)$. So, in the next section, we introduce the algorithms that we developed and their characteristics.

\section{NATURE-INSPIRED ALGORITHMS}

The nature-inspired optimization algorithms have common tasks on their procedures: selection randomly of initial solutions, evaluate the solutions depending on a fitness function, elimination of the worst solutions, and generation of new solutions. This last one is carried out by a set of operations (crossover, mutation, and selection). The significant difference between such algorithms is the generation of new solutions.

In the next subsections, we will show a description of the used algorithms (GA, ES), and their properties are explained by their pseudo-codes.

\subsection{Genetic Algorithm}

Genetic Algorithm (GA) is one of the oldest evolutionary algorithms. It is the most popular EAs in engineering applications. It was developed and created by Holland (1992). It is based on the Darwinian evolution of biological systems. Its operators are the biological operators: crossover, mutation, and selection, which are applied to each population. The population is divided on individuals, where the best individuals have a chance to survive and transfer their characteristics to the next generation. The individual is called chromosome, which presents a solution. In our work, we consider the individual as a real-coded solution (Wright, 1991) presented by a vector and the generation is the iteration to generate a new set of solutions. The procedure is as illustrated in

Algorithm 1:

\begin{tabular}{l}
\hline Algorithm 1: Genetic Algorithm \\
\hline Initialize a solutions randomly; \\
While max_Generation not meet do \\
Evaluate each solution; \\
Rank the solutions; \\
Recombine_BMW pairs of parents; \\
Mutate the offspring by $p_{m} ;$ \\
\hline
\end{tabular}

\section{Replace the worst parents by best offspring;} end while

The operation Evaluate is the evaluation according to its fitness function, where we need to rank the population according to its fitness to perform the Best-Mate-Worst crossover (Abdou and Soltani, 2005; Yeo and Lu, 1999). All the population's individuals are passing on the crossover operation (Recombine_BMW), which is presented by the following expression:

$\left\{\begin{array}{l}x_{i}^{t+1}=\alpha \cdot x_{i}^{t}+(1-\alpha) \cdot x_{j}^{t} \\ x_{j}^{t+1}=\alpha \cdot x_{j}^{t}+(1-\alpha) \cdot x_{i}^{t}\end{array}\right.$

The operation Mutate is a random mutation that is implemented on a few of the offspring (probability of $p_{m}$ ) after the crossover operation. The Gaussian mutation is used as:

$x_{i}^{t+1}=x_{i}^{t+1}+N(0, \sigma)$

Finally, the operator of the selection (Replace) permits to replace the worst individuals of the previous generation by the best individuals of the new generation to keep the size of the population and pass to the next generation. The parameters are shown in Table 2

\subsection{Evolutionary Strategies}

Evolutionary Strategies (ES) is the oldest evolutionary algorithm. It is based on biological evolution. Like the GA, the ES operators are crossover (combination), mutation, and selection (Hansen et al., 2015). Its main advantage is the self-adaptive control of parameters, especially in the mutation task. ES contains many strategies; we use here the $(\mu+\lambda)$ strategy (Abdou and Soltani, 2008) with real-coding individuals. In the selection task, we took $\lambda$ individuals from the offspring's population gathered with the best $\mu$ individuals from the parent's population, and we consider the result as a new generation. Unlike the GA, in the combination (crossover), we don't need to rank the population because the combination is randomly achieved between individuals (a fraction $\lambda$ ). The procedure is illustrated in Algorithm 2:

\begin{tabular}{l}
\hline Algorithm 2: Evolution Strategies \\
\hline Initialize solutions randomly; \\
While max_Generation not meet do \\
Generate new solutions; \\
Recombine pairs of parents; \\
Mutate the offspring; \\
Rank the solutions; \\
Replace new generation $(\mu+\lambda) ;$ \\
end while
\end{tabular}

The crossover task (Recombine) is implemented as in the case of the GA (8) by selecting $\lambda$ parents randomly with a real-coding of the individuals (Wright, 1991). The main characteristic of ES is the self-adaptation mutation. So, we propose to use a log-normal auto-adaptive mutation in Mutate task as:

$\sigma^{t+1}=\sigma^{t} \cdot e^{(\sqrt{n})^{-1} \cdot N(0,1)}$
$x_{i}^{t+1}=x_{i}^{t+1}+N\left(0, \sigma^{t+1}\right)$ 
In the last operation (Replace), we take $\lambda$ individuals of the offspring population and the best $\mu$ ones of parents' population to transfer them to the next generation. The common parameters are max_Generation, which is equal to 20 and population size is equal to 50 . The other parameters are shown in Table 2.

Tab. 2. Parameters setting of different algorithms

\begin{tabular}{|l|l|l|l|}
\hline \multicolumn{2}{|l|}{ GA } & \multicolumn{2}{l|}{ ES } \\
\hline Par. & Val. & Par. & Val. \\
\hline cross. & BMW & $p_{m}$ & 0.3 \\
$\boldsymbol{p}_{\boldsymbol{m}}$ & 0.04 & $\lambda$ & 36 \\
$\boldsymbol{\alpha}$ & $1 / 3$ & $\mu$ & 14 \\
$\boldsymbol{\sigma}$ & 0.04 & $\sigma^{0}$ & 0.3 \\
\hline
\end{tabular}

\section{CONTROLLER DESIGN AND IMPLEMENTATION}

In this section, the only output we consider is the angle $(\theta)$, where the objective is to make the pendulum's orientation null without considering the translational DOF.

\subsection{The closed loop generation}

Before passing to the controller's design phase, we are going to generate the closed-loop system (plant-controller) including the external signals and weighting functions. We notice that the controller has the same structure as the plant (Fig. 3).

The LFT-LPV state space of the augmented plant is as follows:

$\dot{x}=A x+B_{\rho} w_{\rho}+B_{1} w+B_{2} u$

$z_{\rho}=C_{\rho} x+D_{\rho \rho} w_{\rho}+D_{\rho 1} w+D_{\rho 2} u$

$z=C_{1} x+D_{1 \rho} w_{\rho}+D_{11} w+D_{12} u$

$$
w_{\rho}=\Theta z_{\rho}
$$

where $x \in R n$ is the state vector. $u \in R n u$ is the control inputs vector. $y \in R n y$ is the measurement outputs vector. $z \in R n z$ is the controlled outputs. $w \in R n w$ is the exogenous inputs (i.e., disturbances). $z \rho$ and $w \rho \in R r$ are the inputs and outputs of parameter block $\Theta$, with: $\Theta=\operatorname{diag}\left(\rho_{1} \mathrm{I}_{\mathrm{r} 1}, \rho_{2} \mathrm{I}_{\mathrm{r} 2} \ldots \rho_{\mathrm{k}} \mathrm{I}_{\mathrm{rk}}\right)$.

The controller $K(\rho)$ is also an LPV system with LFT representation. We present its state space as follows:

$\dot{x}_{K}=A_{K} x_{K}+B_{K 1} y+B_{K \rho} w_{K \rho}$

$u=C_{K 1} x_{K}+D_{K 11} y+D_{K 1 \rho} w_{K \rho}$

$z_{K \rho}=C_{K \rho} x_{K}+D_{K \rho 1} y+D_{K \rho \rho} w_{K \rho}$

$w_{K \rho}=\Theta z_{K \rho}$

where $x_{K} \in R^{n}$ is the controller states vector. $y \in R^{n y}$ is the measurement outputs from the plant. $u \in R^{n u}$ is the controller outputs. $z_{K \rho}$ and $w_{K \rho} \in R^{r}$ are the inputs and the outputs of the parameter block.

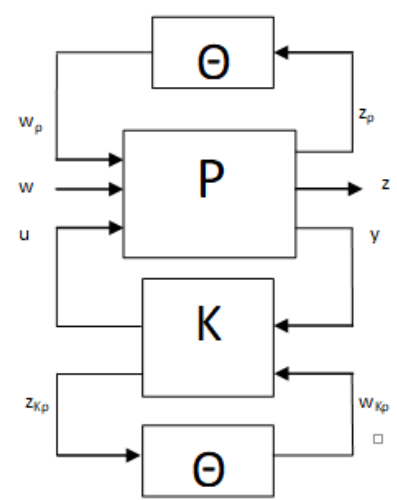

Fig. 3. LFT-LPV closed loop structure

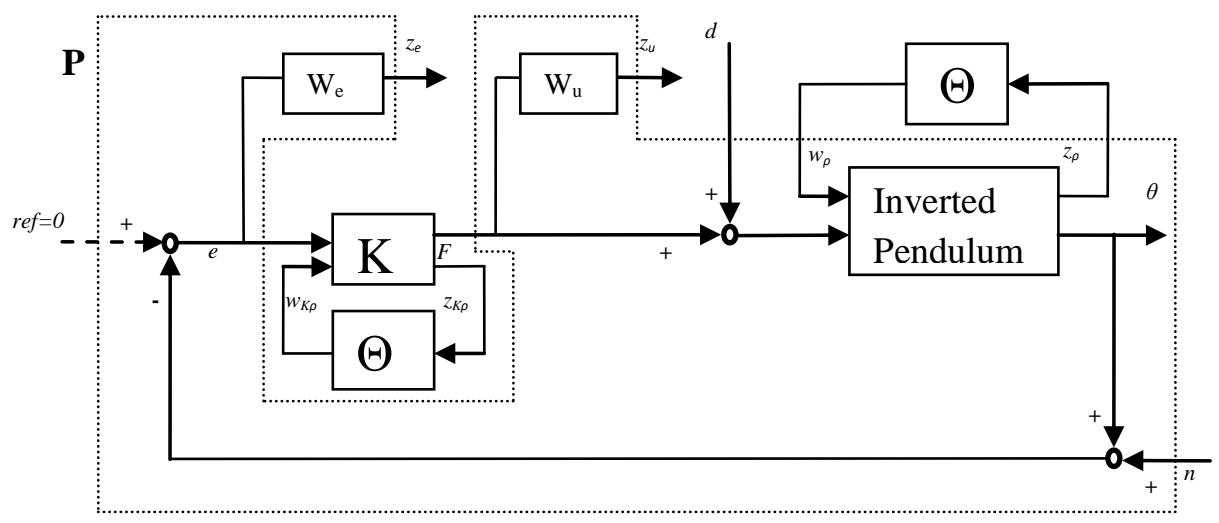

Fig. 4. Closed loop plant-controller interconnection

We interconnect the inverted pendulum system (6)-(7) with the exogenous inputs (disturbance on the input, and measurement noise on the output). We notice in (7) that the output matrix $\left(\mathcal{C}_{1}=\left[\begin{array}{lll}1 & 0 & 0\end{array}\right]\right)$ because we considered the angle $\theta$ as the only output.

As a last step of the designing the closed-loop scheme, we provide the applied force $(F)$ from the generated input $(u)$, from (5):
$F=u-M l \sin (\theta) \dot{\theta}^{2}$

The closed-loop scheme is presented in Fig. 4, in which there are two exogenous inputs (d: disturbance, n: noise), the measurement output is the error, and the only input is the force $\mathrm{F}$. We have two controlled outputs (ze and zu), which present the performance. Because we have four varying parameters (Tab. 1) $z_{\rho}$, $w_{\rho}, z_{K \rho}$, and $w_{K \rho} \in R^{4}$. 
As we noticed previously, to design the controller, we have to introduce the weighting functions' parameters. Our contribution is based on the choice of the weighting functions' parameters by two evolutionary algorithms (GA and ES) to get the optimal desired performance, and as a consequence, a robust scheduled controller that guarantees this performance. In the next subsection, we show the optimization procedure.

\subsection{The optimization procedure}

The optimization tools, GA and ES, need to specify some characteristics. The weighting functions formula has been taken as a first-order filter. The performance criterion is presented by $W_{e}$ and $W_{u}$ (Fig. 4).

$W_{e}=k_{e} \frac{\tau_{e 1} s+1}{\tau_{e 2} s+1}$
$W_{u}=k_{u} \frac{\tau_{u 1} s+1}{\tau_{u 2} s+1}$

Therefore, each solution contains six parameters:

sol. $=\left[\begin{array}{llllll}k_{e} & \tau_{e 1} & \tau_{e 2} & k_{u} & \tau_{u 1} & \tau_{u 2}\end{array}\right]$

The main effect of the optimization by the evolutionary algorithms is the multi-objective. We have taken two dynamical indices; settling time $\operatorname{ST}(\theta(t))$, and the overshoot $O V(\theta(t))$. By experience, the system (inverted pendulum on a cart) needs big force to stabilize in a short time, it could be faced with a big overshoot and oscillations (Fiacchini et al., 2006; Raffo et al., 2007; Hasseni and Abdou, 2017). So, it makes sense to give a small weight to the settling time and a big weight to the overshoot. We have chosen the fitness as follows:

$$
\text { Fitness }=0.3 S T(\theta(t))+0.7 \mathrm{OV}(\theta(t))
$$

The controller synthesis algorithm is achieved by the small gain theorem via LMI conditions. This approach is developed in (Packard, 1994; Apkarian and Gahinet, 1995) and we have been helped by the toolbox LPVTools (Hjartarson et al., 2015).

As we noticed, the optimization problem has been achieved by two algorithms (GA and ES). Algorithm 3 presents the optimization algorithm. We note Algorithm_ $i$ to mean Algorithm 1 and Algorithm 2 because we use both of them to solve the same problem. The details of their procedures and characteristics are mentioned in Section 3 and Table 2.

\begin{tabular}{l}
\hline Algorithm 3 : Optimal weighting functions \\
\hline Initialize a solutions (17) randomly; \\
While max_Generation not meet do \\
Generate the closed loop (Fig. 4); \\
Design the own controller; \\
Evaluate the solutions based on fitness (18) \\
Replace the solutions based on Algorithm_i; \\
end while
\end{tabular}

The optimal parameters that are obtained by GA and ES are presented in Table 3. Thanks to the optimization algorithms we guarantee the low pass filter of the control on both algorithms because the $\mathrm{H}_{\infty}$ controller guarantees this condition $\left|\frac{K}{1+K P}\right| \leq$ $\left|\frac{1}{W_{u}}\right|$ where $K$ is the controller and $P$ is the plant (Zhou and Doyle, 1998).
Tab. 3. Weighting functions parameters

\begin{tabular}{|l|l|l|l|l|l|l|}
\hline Parameter & $\boldsymbol{k}_{\mathrm{e}}$ & $\boldsymbol{\tau}_{\mathrm{e} 1}$ & $\boldsymbol{\tau}_{\mathrm{e} 2}$ & $\boldsymbol{k}_{u}$ & $\boldsymbol{\tau}_{\mathrm{u} 1}$ & $\boldsymbol{\tau}_{\mathrm{u} 2}$ \\
\hline LPV-GA & 3.286 & 0.4015 & 6.603 & 0.673 & 6.362 & 0.242 \\
\hline LPV-ES & 6.165 & 7.493 & 2.772 & 1.091 & 5.968 & 0.645 \\
\hline $\begin{array}{l}\text { (Hasseni and } \\
\text { Abdou, 2017) }\end{array}$ & 5 & 0.04 & 1 & 1 & 1 & 0.1 \\
\hline
\end{tabular}

By taking the guidelines of Skogestad and Postlethwaite (2003) about the filters' shaping, we can find that the obtained controller with GA (LPV-GA) is suitable with these guidelines. The inverse of sensitivity function $\left(1 / W_{e}\right)$ is a high pass filter with a small gain in low frequencies and a big gain in high frequencies. The desired $\left|\frac{K}{1+K P}\right|=\left(1 / W_{u}\right)$, should be a low-frequency function with the maximum gain in low frequencies, while the highfrequency gain is limited by the controller bandwidth. The equations (19)-(20) present the standard forms weights $W_{e}$ and $W_{u}$ of the LPV-GA controller, presented in Table 3.

$W_{e}=\frac{1 / M_{s} s+\omega_{b}}{s+\varepsilon \omega_{b}}=\frac{1 / 5.05}{s+0.4977}$

$W_{u}=\frac{s+{ }^{\omega_{b c}} / M_{u}}{\varepsilon_{u} s+\omega_{b c}}=\frac{s+0.233 / 1,485}{0.0565 s+0.233}$

Fig. 5 presents the bode magnitude of the inverse filters (19)(20). The inverse of the sensitivity function $\left(1 / W_{e}\right)$ is a high pass filter, the steady-state error to step input $(\varepsilon=0.304)$, the overshoot magnitude (peak sensitivity, $M_{s}=5.05$ ), and limit closedloop bandwidth $\left(\omega_{b}=0.4977 \mathrm{rad} / \mathrm{s}\right)$. The maximum gain of $\left(1 / W_{u}\right)$ is fairly large $\left(M_{u}=1.485\right)$, it is limited by the controller bandwidth $\left(\omega_{b c}=0.233 \mathrm{rad} / \mathrm{s}\right)$. To make $W_{u}$ proper, we introduce a faraway pole, as an obtained result, $\varepsilon_{1}$ is very small $\left(\varepsilon_{1}=\right.$ 0.0565).

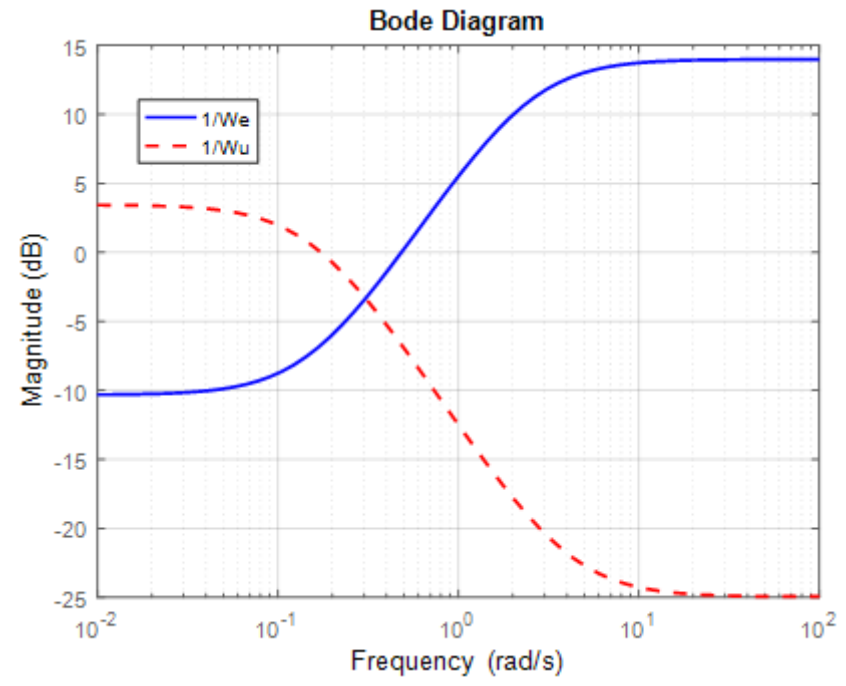

Fig. 5. Performance weight (solid) and control weight (dashed) of LPV-GA

\subsection{Simulation results}

This table presents the nominal values of the inverted pendulum on the cart used in this paper. 
Seif-El-Islam Hasseni, Latifa Abdou

Robust LFT-LPV H${ }_{\infty}$ Control of an Underactuated Inverted Pendulum on a Cart with Optimal Weighting Functions Selection by GA and ES

Tab. 4. Nominal parameters of the vehicle

\begin{tabular}{|l|l|l|l|}
\hline Parameter & Description & Value & Unit \\
\hline $\boldsymbol{m}$ & Cart's mass & 35 & $\mathrm{Kg}$ \\
\hline $\boldsymbol{M}$ & Pendulum's mass & 70 & $\mathrm{Kg}$ \\
\hline $\boldsymbol{I}$ & Pendulum's arm length & 1 & $\mathrm{~m}$ \\
\hline $\boldsymbol{g}$ & Gravity constant & 9.8 & $\mathrm{~m} . \mathrm{s}^{-2}$ \\
\hline $\boldsymbol{b}$ & Friction coefficient & 40 & $\mathrm{~N} . \mathrm{s} . \mathrm{m}^{-1}$ \\
\hline
\end{tabular}

We have gotten an LFT-LPV $\mathrm{H}_{\infty}$ controller with weighting functions' selection by GA optimization and another by ES. Fig. 6 presents a comparison of the dynamic response and the input between the three LFT-based LPV controllers; the obtained controller with GA (LPV-GA), the obtained one with ES (LPV-ES), and the existing one (Hasseni and Abdou, 2017) (LFT-LPV). Besides, we also show the gridding LPV technique and polytopic LPV technique (Briat, 2015). In this simulation, the performances of each method are presented in Fig. 6 and Table 5, where the initial angle is $60^{\circ}$. Among these controllers, the best performance is obtained by the LPV-GA algorithm.

Tab. 5. Performances comparison between different LPV techniques

\begin{tabular}{|c|c|c|c|}
\hline Technique & Reference & $\begin{array}{c}\text { Overshoot } \\
(\%)\end{array}$ & $\begin{array}{c}\text { Settling time } \\
\text { (s) }\end{array}$ \\
\hline Polytopic-LPV & $\begin{array}{c}\text { (Robert et al., } \\
\text { 2010) }\end{array}$ & 18 & 3 \\
\hline Gridding-LPV & $\begin{array}{c}\text { (Hjartarson et } \\
\text { al., 2015) }\end{array}$ & 17 & 6.45 \\
\hline LFT-LPV & $\begin{array}{c}\text { (Hasseni and } \\
\text { Abdou, 2017) }\end{array}$ & 7.2 & 1.47 \\
\hline LPV-ES & This work & 10.7 & 0.6 \\
\hline LPV-GA & This work & 7.6 & 0.53 \\
\hline
\end{tabular}
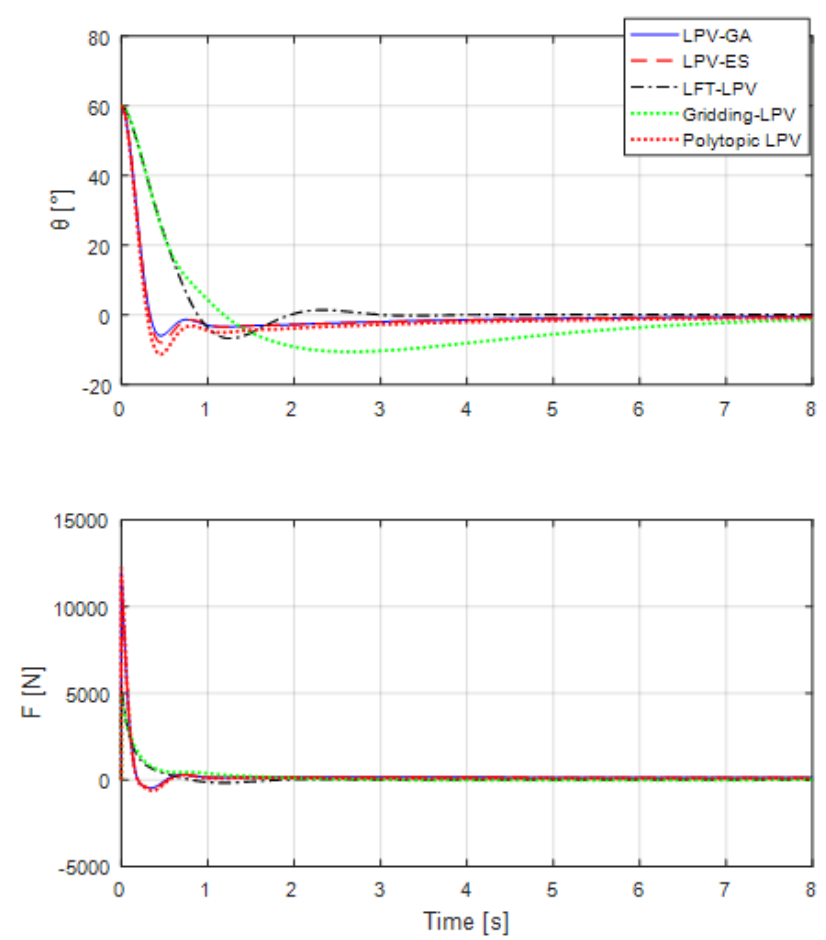

Fig. 6. Dynamic response comparison between different LPV techniques
To demonstrate that the proposed LPV method indeed increases the region of stability, Fig. 7 presents a test of different initial angles with two controllers; the existing controller called (LFT-LPV) and the optimal one gotten by GA called (LPV-GA). It must be shown that the existing controller (dashed line) could not exceed $75^{\circ}$ as initial angle. With a bigger initial angle than $75^{\circ}$, the system is completely unstable. On the other hand, with the proposed method (solid line), the system is stable till $86^{\circ}$ as an initial angle.
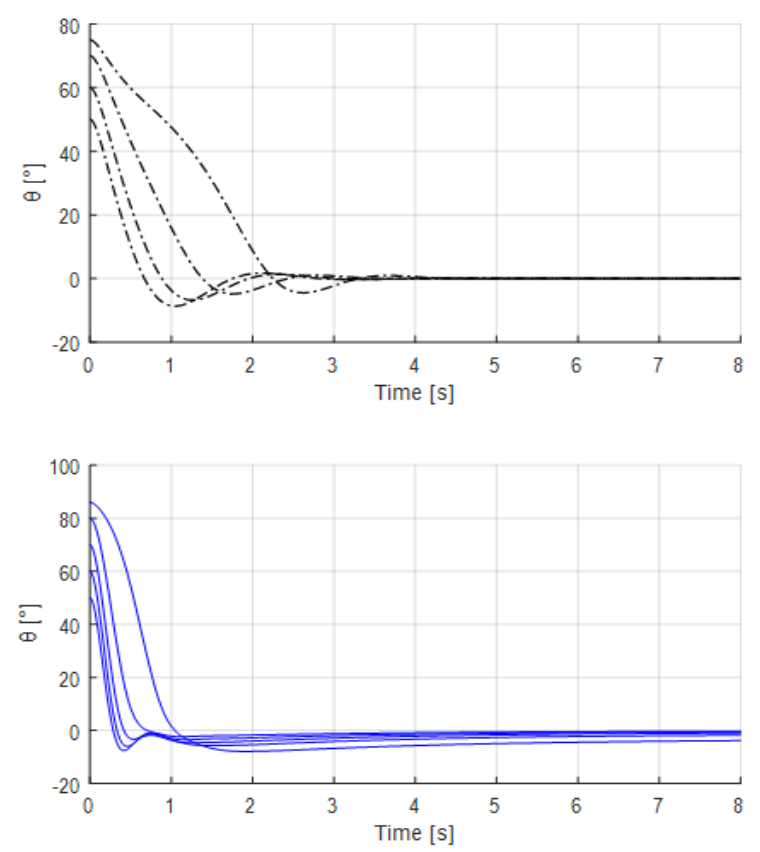

Fig. 7. The angle responses with different initial angles, LPV-GA (solid) and LFT-LPV (dashed)

Now we pass to another point, the robustness to the exogenous inputs. The external inputs we choose are the input disturbance and the noise. As shown in Fig. 8, we apply a permanent noise between $\pm 2^{\circ}$. Starting from $5 \mathrm{~s}$, we apply a disturbance force of $200 \mathrm{~N}$ as shown in Fig. 8.

Remark 1: In the simulation, we don't apply the robust LPV controller on the approximated LPV model (6)-(7), but on the original nonlinear model itself (3).

Fig. 9 presents a comparison of the angle response $(\theta)$, the linear velocity $(V)$, and the input $(F)$ between $L P V-G A$ and $L P V-E S$ with the presence of disturbance and noises. In the curve of $(\theta)$, we notice that there is robustness to the noises. We notice also that there is a variation in $5 s$ due to the suddenly applied force, but the disturbance is ultimately rejected.

We conclude that the nature-inspired optimization tools, GA and ES, helped us to choose the suitable weighting functions parameters to get good performance. Also, the scheduled LFT-LPV controller guarantees the robustness against the external signals. But, the linear velocity $(V)$ has not reached the null at the same time with the angle (the vehicle has not stopped) because we didn't care for the velocity in the controller design. The system is considered as a Single-Input Single-Output system (SISO). This is what we are discussing in the next section. 

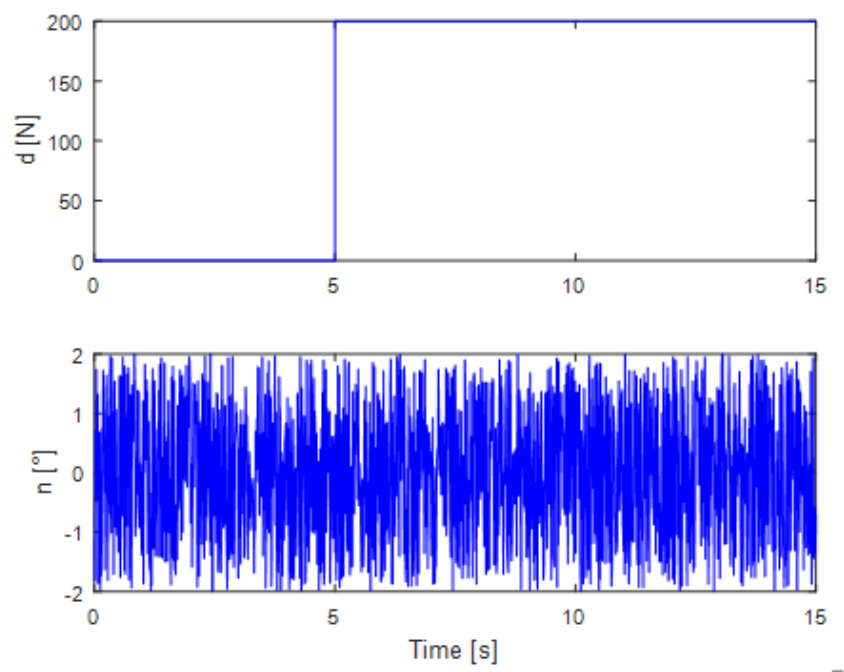

Fig. 8. The disturbance and the noise
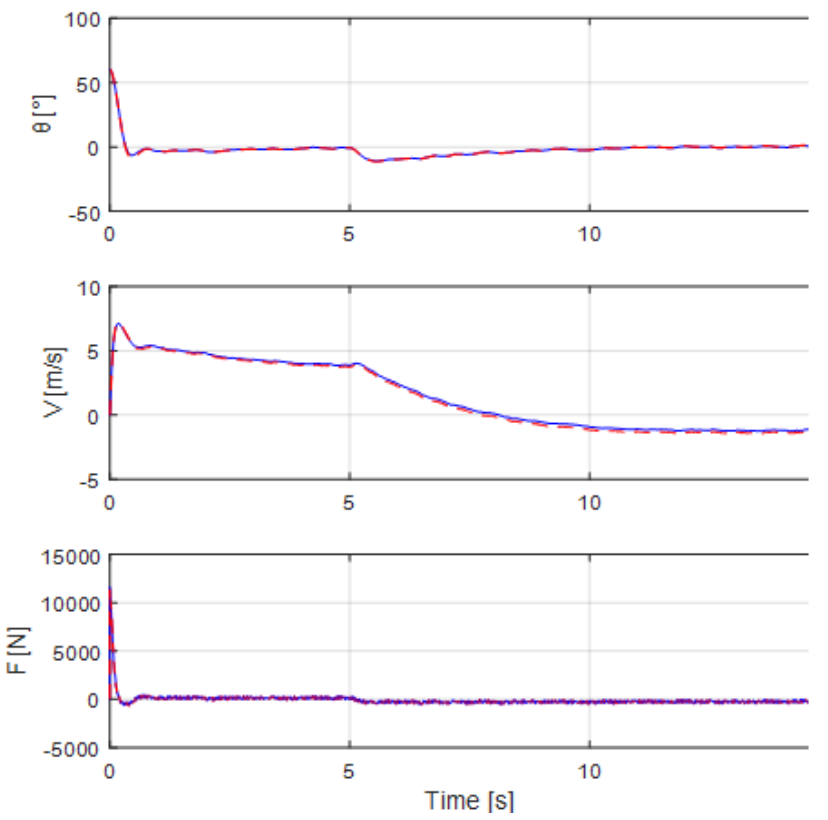

Fig. 9. The angle $(\theta)$, the velocity $(V)$ and the force $(F)$ responses with presence of disturbance and noise

\section{EXTENDED LPV STABILIZATION TO THE 2DOF}

An underactuated mechanical system (UMS) is a system that has fewer control inputs than the degrees of freedom, as well as the inverted pendulum. It has two degrees of freedom; one is relating to translational coordinate $(x)$, and the other is relating to rotational coordinate $(\theta)$, but just one control input, the force $(F)$. In Section 4, the inverted pendulum has been stabilized by a robust LPV $\mathrm{H}_{\infty}$ controller by considering it as a SISO system. In the previous simulation (Section 4), the velocity has reached the null very slowly (300 seconds).

We have to impose the constraint of the underactuated characteristic into account. One may say that alternatively to consider the angle $(\theta)$ as the only output, we consider two outputs: the angle $(\theta)$ and the linear velocity $(\dot{x})$ and generate its suitable LPV controller.
We have tried to control the inverted pendulum as a general Single-Input Multi-Outputs system (SIMO) by changing the output matrix of $(6)$ by $\left(\mathcal{C}_{1}=\left[\begin{array}{lll}1 & 0 & 0 \\ 0 & 0 & 1\end{array}\right]\right)$. The response, either the angle or the velocity is oscillation and never stabilize (Fig. 10). In this test, the weighting functions are obtained by GA.
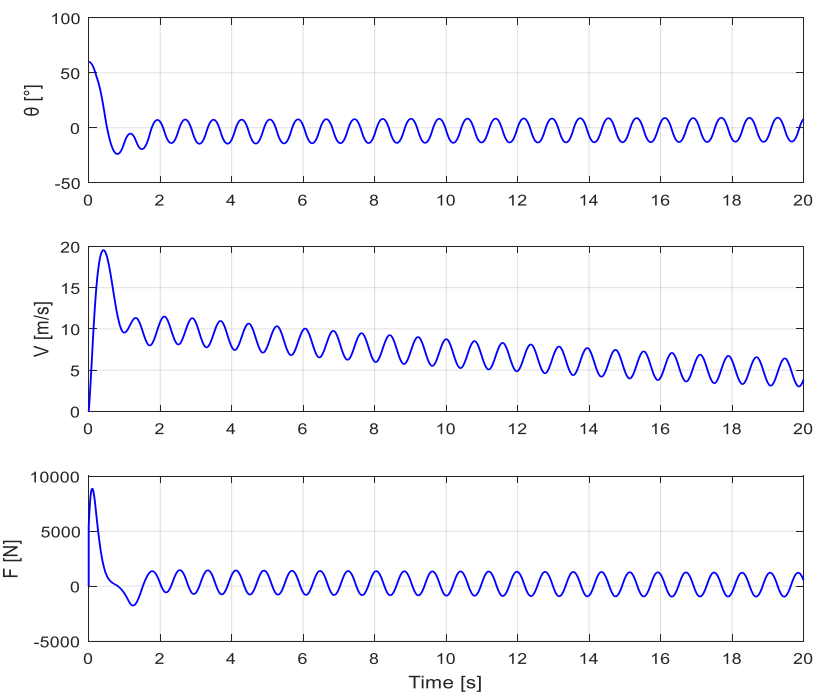

Fig. 10. The dynamic response of the inverted pendulum as a SIMO system

In literature, there is no unique methodology to control a UMS. There are different classifications. One of the most common classifications is Seto and Baillieul's. It depends on the so-called Control Flow Diagram (CFD). According to this classification, a UMS could have a chain structure, tree structure, or isolatedvertex structure (Seto and Baillieul, 1994; Choukchou-Braham et al., 2014a). The inverted pendulum is found under the tree class.

There are sub-classes into the tree structure (A1, A2) (Choukchou-Braham et al., 2014a). Since the first (A1) could be converted to a chain class by changing the states and controlled as a chain structure by Backstepping (Rudra et al., 2017). The other subclass (A2) could not. In (Choukchou-Braham et al., 2014b), the authors have proposed a method where the configuration variables (the degrees of freedom) of the system were controlled simultaneously and this is what we are going to develop. We have to include a term related to stabilize the angle $(\theta)$ and another term related to stabilize the linear velocity $(\dot{x})$ in the control law. We will denote to the state $\dot{x}$ by $v$.

The only expression that has information about $(\theta)$ is the error (the measurement output of the LPV plant, Fig. 4). The closedloop system has been changed by adding a proportional term related to $(v), k_{v} v$ (Fig. 11). The gain $k_{v}$ presents the weight of the velocity, and we choose $k_{v}=0.02$. The new output is $(\theta+0.02 v)$.

Remark 2: In this stage, we didn't design another controller, but we kept the controller we have designed in Section 4.

We pass now to the simulation phase. Fig. 12 presents the dynamic response of the angle $(\theta)$, the velocity $(V)$, and the force $(F)$ of both controllers (LPV-GA and LPV-ES) with the presence of disturbance and noise as well as presented in Fig. 8. In addition to the angle, the velocity is controlled and it has reached zero on 


\section{sciendo}

Seif-El-Islam Hasseni, Latifa Abdou

Robust LFT-LPV H ${ }_{\infty}$ Control of an Underactuated Inverted Pendulum on a Cart with Optimal Weighting Functions Selection by GA and ES

both curves (LPV-GA and LPV-ES). The overshoot exceeds ($\left.20^{\circ}\right)$, and that is what the pendulum needs to balance itself. We note also that the system is robust against the permanent noise and the disturbance.

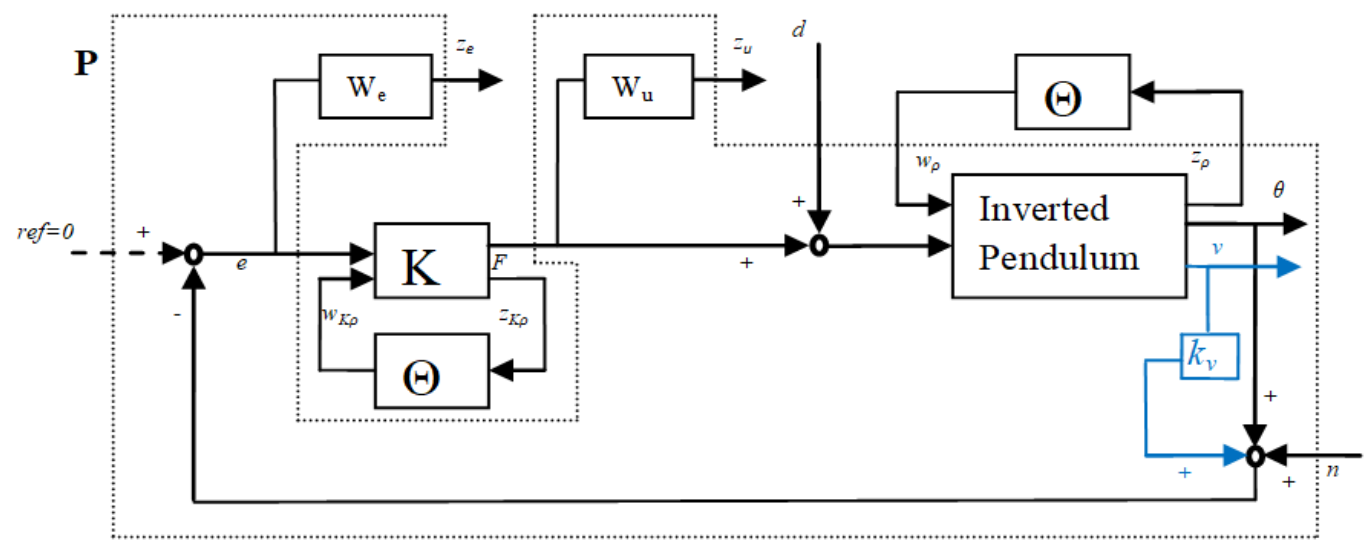

Fig. 11. Underactuated closed loop plant-controller
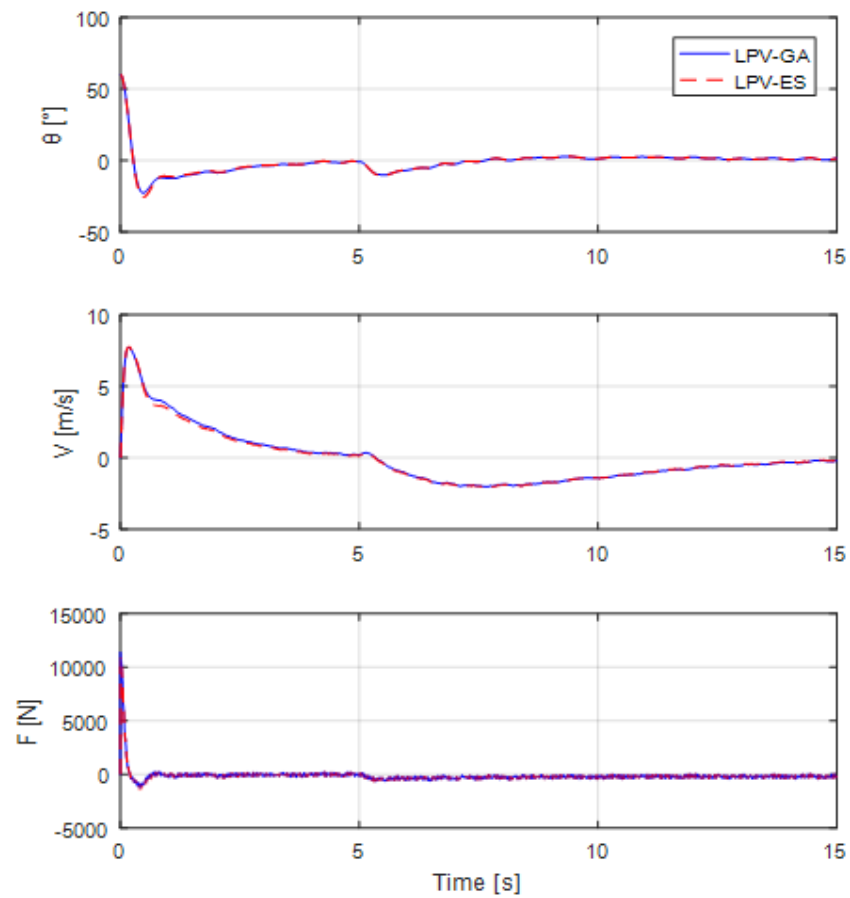

Fig. 12. The angle $(\theta)$, the velocity $(V)$ and the force $(F)$ responses with adding the velocity term

We have to mention that when the $\mathrm{H}_{\infty}$ controller is achieved, it so guarantees the worst case of uncertainties. That means the closed-loop system is asymptotically stable for any $\|\Delta\|_{\infty} \leqslant 1$.

Remark 3: the stability of the augmented system is robust (plant-weighting functions), so, the performance of the system is robust because the performance is presented by the weighting functions.

In our system, the pendulum's mass presents the mass of the rider. We can apply the uncertainty according to the rider's mass by taking $70 \mathrm{Kg}$ as a nominal mass (Tab. 4). In this simulation, we have taken $M=$ nominal value $\pm 43 \%(40 \mathrm{Kg}, 70 \mathrm{Kg}$, and $100 \mathrm{Kg})$. Fig. 13 presents the response with the uncertainty of $L P V-G A$ and Fig. 14 presents the response with the uncertainty of $L P V-E S$. We note that the controllers are robust to the uncertainty, the angular overshoot is between $-20^{\circ}$ and $-30^{\circ}$, the peak velocity is $8 \mathrm{~m} / \mathrm{s}$ when $(M=100), 9 \mathrm{~m} / \mathrm{s}$ when $(M=70)$ and $10 \mathrm{~m} / \mathrm{s}$ when $(M=40)$.

All the previous simulations (Fig. 13 and Fig. 14) are achieved with $60^{\circ}$ as an initial angle. In Fig. 15 and Fig. 16, the simulation results of the dynamic responses are achieved with different initial angles $\left(60^{\circ}, 30^{\circ},-30^{\circ}\right.$ and $\left.-60^{\circ}\right)$. Fig. 15 presents the dynamic responses of $L P V-G A$ and Fig. 16 presents the dynamic responses of LPV-ES. On both controllers, the 2DOF are stabilized. The angle and velocity have reached the equilibrium point simultaneously. Besides, it is robust, whatever the initial angle.
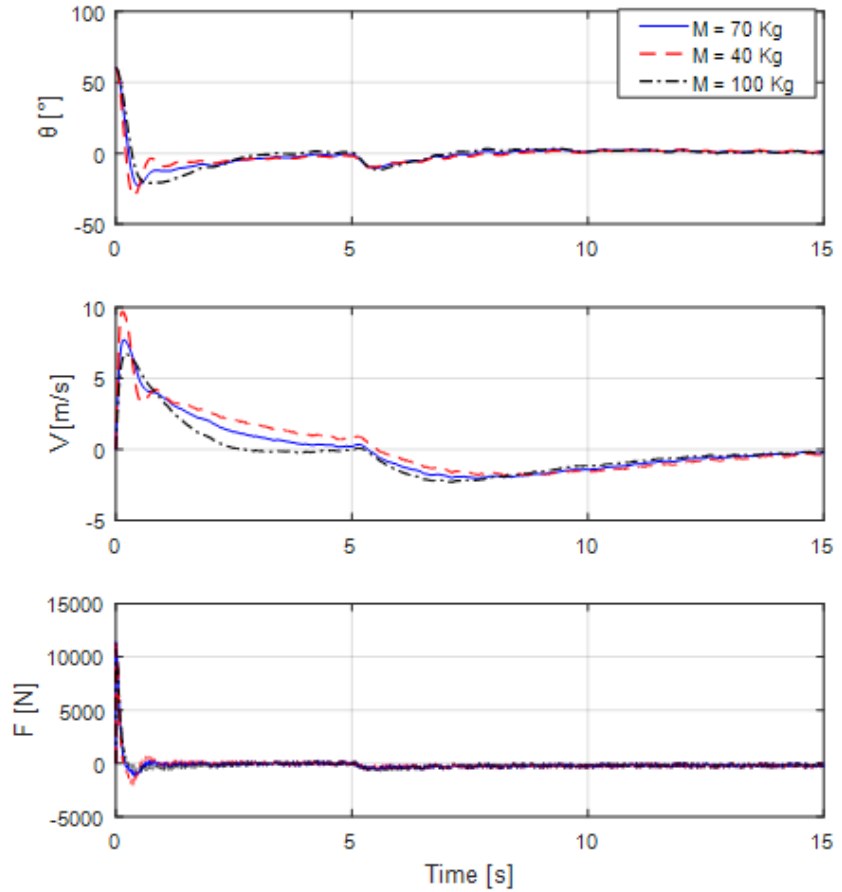

Fig. 13. The angle $(\theta)$, the velocity $(V)$ and the force $(F)$ responses with uncertainty on $L P V-G A$ 

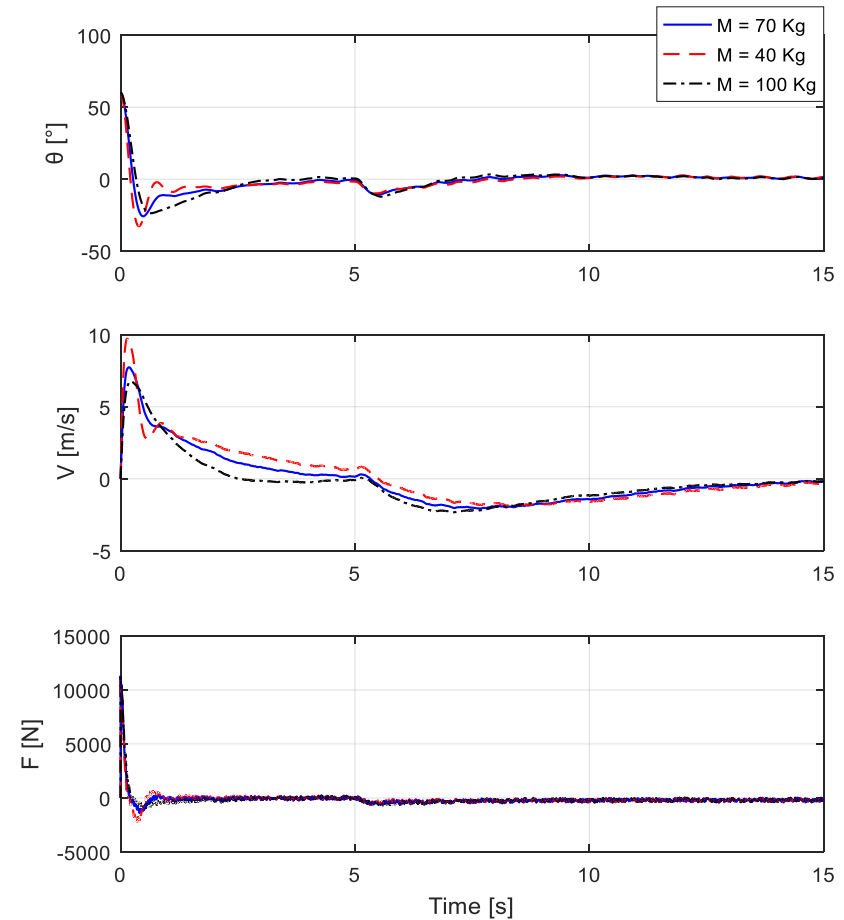

Fig. 14. The angle $(\theta)$, the velocity $(V)$ and the force $(F)$ responses with uncertainty on $L P V-E S$
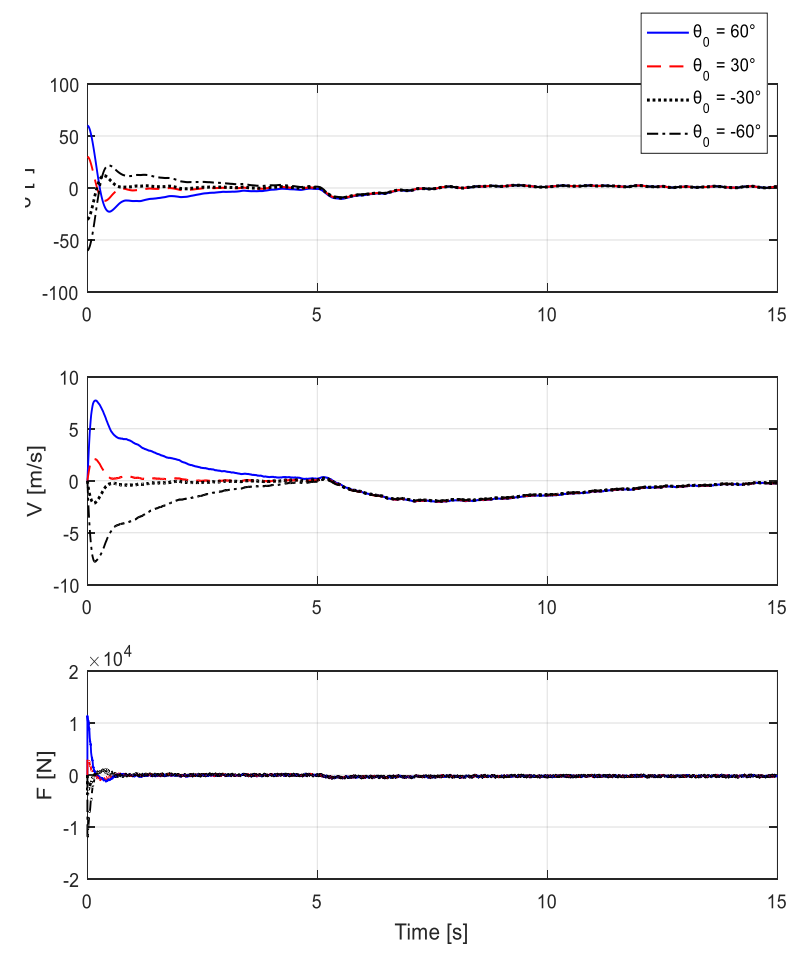

Fig. 15. The angle $(\theta)$, the velocity $(V)$ and the force $(F)$ responses with different initial angles on LPV-GA
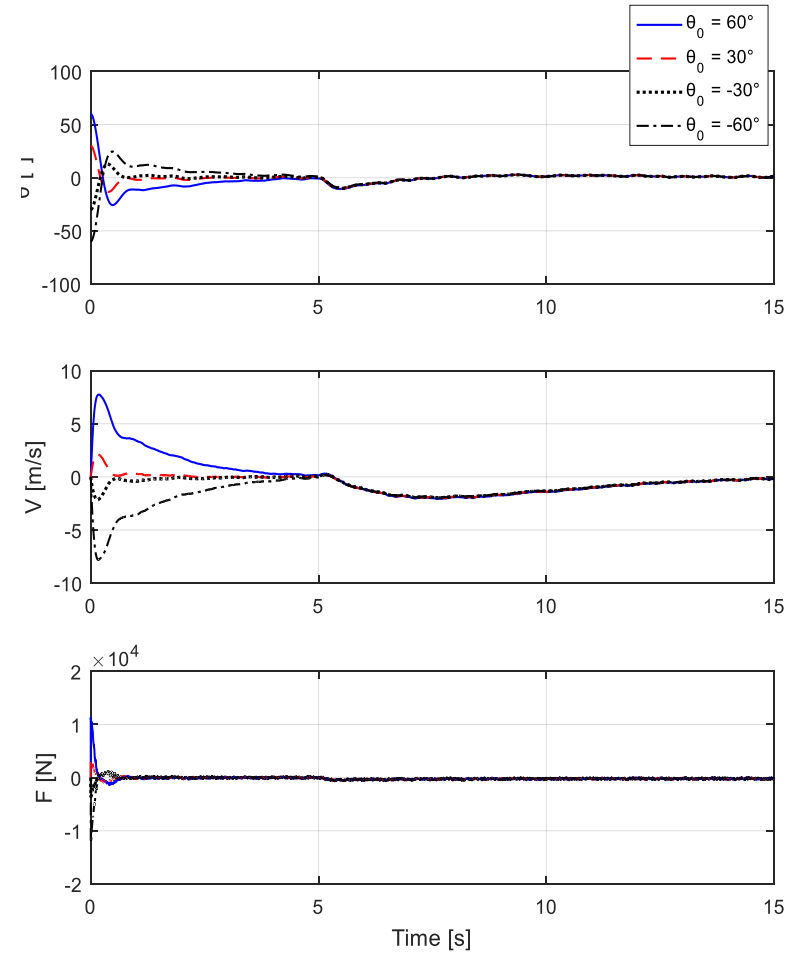

Fig. 16. The angle $(\theta)$, the velocity $(V)$ and the force $(F)$ responses with different initial angles on LPV-ES

\section{CONCLUSION}

In this paper, the robust stabilization problem of the inverted pendulum on a cart is considered. We have applied a scheduled robust controller with type LFT-LPV $\mathrm{H}_{\infty}$. As a first step, we have developed its LFT-LPV representation from the nonlinear model. Second, we have focused on the weighting functions' parameters selection. We have suggested solving it through two natureinspired optimization algorithms (GA and ES). Thanks to those, besides to get optimal performance, the robustness is guaranteed against the disturbances and the uncertainties. The last and important point we have investigated is the problem of underactuation. The inverted pendulum imposes this constraint and our objective is to balance the inverted pendulum where its angle and velocity should be zero without adding another actuator. After we modify the closed-loop structure, the simulation results have shown an optimal performance, the objective is successfully achieved. It is robust against external signals and uncertainties.

\section{REFERENCES}

1. Abbas H.S., Tóth R., Petreczky M., Meskin N., and Mohammadpour J. (2014), Embedding of nonlinear systems in a Linear Parameter-Varying representation, In: Proc. of IFAC World Congress, Cape Town, South Africa, 6907-6913.

2. Abdou L., and Soltani F. (2005), OS-CFAR and CMLD threshold optimization with genetic algorithms, In: Proc. of $3^{\text {rd }}$ International Conference on Systems, Signals \& Devices, Vol III Communication and Signal Processing, Sousse, Tunisia.

3. Abdou L., and Soltani F. (2008), OS-CFAR and CMLD threshold optimization in distributed systems using evolotionary strategies, Signal, Image and Video Processing, Vol. 2, No. 2, 155-167. 
4. Alcalá E., Puig V., Quevedo J., and Rosolia U. (2020), Autonomous racing using Linear Parameter Varying-Model Predictive Control (LPV-MPC), Control Engineering Practice, Vol. 95, 104270.

5. Alfaro-Cid E., McGookin E.W., and Murray-Smith D.J. (2008), Optimisation of the weighting function of an $\mathrm{H}_{\infty}$ controller using genetic algorithms and structured genetic algorithms, International Journal of Systems Science, Vol. 39, No. 4, 335-347.

6. Apkarian P., and Gahinet P. (1995), A convex characterization of gain-scheduled $\mathrm{H}_{\infty}$ controller, IEEE Transactions on Automatic Control, Vol. 40, No. 7, 853-864.

7. Beaven R.W., Wright M.T., and Seaward D.R. (1996), Weighting function selection in the $\mathrm{H}_{\infty}$ design process, Control Engineering Practice, Vol. 4, No. 7, 625-633.

8. Boubaker 0. (2013), The inverted pendulum benchmark in nonlinear control theory: A survey, International Journal of Advanced Robotic Systems, Vol. 10, No. 5, 233-241.

9. Briat C. (2015), Linear Parameter-Varying and Time-Delay Systems: Analysis, Observation, Filtering \& Control, Springer-Verlag, Heidelberg.

10. Choukchou-Braham A., Cherki B., Djemaï M., and Busawon K (2014a), Classification of Underactuated Mechanical Systems, In: Choukchou-Braham A., Cherki B., Djemaï M., and Busawon K. (eds.), Analysis and Control of Underactuated Mechanical Systems, Springer, London, 35-54.

11. Choukchou-Braham A., Cherki B., Djemaï M., and Busawon K. (2014b), Control Design Schemes for Underactuated Mechanical Systems, In: Choukchou-Braham A., Cherki B., Djemaï M., and Busawon K. (eds.), Analysis and Control of Underactuated Mechanical Systems, Springer-Verlag, London, 55-91.

12. Do A.L., Sename O., Dugard L., and Soualmi B. (2011), Multiobjective optimization by genetic algorithms in $\mathrm{H}_{\infty} / \mathrm{LPV}$ control of semi-active suspension, In: Proc. of IFAC World Congress, Milano, Italy, 7162-7167.

13. El-Bardini M., and El-Nagar A.M. (2014), Interval type-2 fuzzy PID controller for uncertain nonlinear inverted pendulum system, ISA Transactions, Vol. 53, 732-743.

14. Fiacchini M., Viguria A., Cano R., Prieto A., Rubio F.R., Aracil J., and Canudas-de-Wit C. (2006), Design and experimentation of a personal pendulum vehicle, In: Proc. of Portuguese Conference on Automatic Control, Lisbona, Portugal.

15. Hansen N., Arnold D.V., and Auger A. (2015), Evolution Strategies, In: Kacprzyk J., and Pedrycz W. (eds.), Springer Handbook of Computational Intelligence, Springer, Heidelberg, 871-898.

16. Hasseni S., and Abdou L. (2017), Robust LPV control applied to a personal pendulum vehicle, In: Proc. of International Conference on Sciences and Techniques of Automatic Control and Computer Engineering, Monastir, Tunisia, 6-11.

17. Hasseni S., and Abdou L. (2018), Integral backstepping/LFT-LPV $\mathrm{H}_{\infty}$ control for the trajectory tracking of a quadcopter, In: Proc. of International Conference on Systems and Control, Valencia, Spain, 348-353.

18. Hasseni S., and Abdou L. (2020), Robust LPV control for attitude stabilization of a quadrotor helicopter under input saturations, $\mathrm{Ad}$ vances in Technology Innovation, Vol. 5, No. 2, 98-111.

19. Hjartarson A., Seiler P., and Packard A. (2015), LPV Tools: a toolbox for modeling, analysis and synthesis of parameter varying control systems, IFAC PapersOnLine, Vol. 48, No. 26, 136-145.

20. Holland J.H. (1992), Adaptation in Natural and Artificial Systems, MIT Press, MA, USA

21. Hu J., Bohn C., and Wu H.R. (2000), Systematic $H_{\infty}$ weighting function selection and its application to the real-time control of a vertical take-off aircraft, Control Engineering Practice, Vol. 8, No. 3 , 241-252.

22. Iwasaki T., and Shibata G. (2001), LPV system analysis via quadratic separator for uncertain implicit system. IEEE Transactions on Automatic Control, Vol. 46, No. 10, 1195-1208.
23. Li S., Jiang S., and Pan F. (2019), Event-triggered fault detection for networked LPV systems, Circuits, Systems, and Signal Processing, Vol. 38, No. 7, 2992-3019.

24. Liu T.J., Du X., Sun X.M., Richter H., and Zhu F. (2019), Robust tracking control of aero-engine rotor speed based on switched LPV model, Aerospace Science and Technology, Vol. 91, 382-390.

25. Liu Z., Theilliol D., Gu F., He Y., Yang L., and Han J. (2017), State feedback controller design for affine parameter-dependent LPV systems, IFAC PapersOnLine, Vol. 50, No. 1, 9760-9765.

26. López-Estrada F.R., Ponsart J.C., Theilliol D., Zhang Y., and Astorga-Zaragoza C.M. (2016), LPV model-based tracking control and robust sensor fault diagnosis for a quadrotor UAV, Journal of Intelligent \& Robotic Systems, Vol. 84, 163-177.

27. Nguyen A.T., Chevrel P., and Claveau F. (2020), LPV static output feedback for constrained direct tilt control of narrow tilting vehicles, IEEE Transactions on Control Systems Technology, Vol. 28, No. 2, 661-670

28. Ohhira T., and Shimada A. (2017), Model predictive control for an Inverted pendulum robot with time-varying, IFAC PapersOnLine, Vol. 50 , No. 1, 776-781.

29. Packard A. (1994), Gain scheduling via linear fractional transformations, Systems and Control Letters, Vol. 22, No. 2, 79-92.

30. Park M.S., and Chwa D. (2009), Swing-up and stabilization control of inverted-pendulum systems via coupled sliding-mode control method, IEEE Transactions on Industrial Electronics, Vol. 56, No. 9 , 3541-3555.

31. Prasad L.B., Tyagi B., and Gupta H.O. (2014), Optimal control of nonlinear inverted pendulum system using PID controller and LQR: Performance analysis without and with disturbance input, International Journal of Automation and Computing, Vol. 11, No. 6, 661670.

32. Raffo G.V., Ortega M.G., and Rubio F.R. (2007), Nonlinear $\mathrm{H}_{\infty}$ Control Applied to the Personal Pendulum Car, In: Proc. of European Control Conference, Kos, Greece, 2065-2070.

33. Rechenberg I. (1973), Evolutionstrategie: Optimieruna Technischer Systeme nach Prinzipien der Biologischen Evolution, FrommannHolzboog-Verlag, Stuttgart, Germany.

34. Robert D., Sename O., and Simon D. (2010), An $H_{\infty}$ LPV design for sampling varying controllers: experimentation with a T-inverted pendulum, IEEE Transactions on Control Systems Technology, Vol. 18, No. 3, 741-749.

35. Rotondo D., Cristofaro A., Johansen T.A., Nejjari F., and Puig V. (2018), Diagnosis of icing and actuator faults in UAVs using LPV unknown input observers, Journal of Intelligent \& Robotic Systems, Vol. 91, №. 3-4, 651-665.

36. Rudra S., Barai R.K., and Maitra M. (2017), Block Backstepping Control of the Underactuated Mechanical Systems, In: Rudra S., Barai R.K., and Maitra M. (eds.), Block Backstepping Design of Nonlinear State Feedback Control Law for Underactuated Mechanical Systems, Springer-Verlag, Singapore, 31-52.

37. Salhi S., Aouani N., and Salhi S. (2015), LPV affine modeling, analysis and simulation of DFIG based wind energy conversion system, In: Proc. of International Conference on Modelling, Identification and Control, Sousse, Tunisia

38. Scherer C.W. (2001), LPV control and full block multipliers, Automatica, Vol. 37, No. 3, 361-375.

39. Seto D., and Baillieul J. (1994), Control problem in super-articulated mechanical systems, IEEE Transactions on Automatic Control, Vol. 39, No. 14, 2442-2453.

40. Shamma J., and Athans M. (1991), Gain scheduling: potential hazards and possible remedies, In: Proc. of American Control Conference, Boston, USA, 516-521.

41. Siradjuddin I., Amalia Z., Setiawan B., Ronilaya F., Rohadi E., Setiawan A., Rahmad C., and Adhisuwignjo S. (2018), Stabilising a cart inverted pendulum with an augmented PID control scheme, MATEC Web of Conference, Vol. 197, 11013.

42. Skogestad S., and Postlethwaite I. (2003), Multivariable Feedback Control, Analysis and Design, John Wiley \& Sons, Chichester. 
43. Tasoujian S., Salavati S., Franchek M.A., and Grigoriadis K.M. (2020), Robust delay-dependent LPV synthesis for blood pressure control with real-time Bayesian parameter estimation, IET Control Theory \& Applications, Vol. 14, No. 10, 1334-1345.

44. Vu V.T., Sename O., Dugard L., and Gaspar P. (2017), Multi objective $\mathrm{H}_{\infty}$ active-roll bar control for heavy vehicles, IFAC PapersOnLine, Vol. 50, No. 1, 13802-13807.

45. Wright A. (1991), Genetic Algorithms for Real Parameter Optimization, Morgan Kaufmann, San Mateo, California.

46. Wu F. (2001), A generalized LPV system analysis and control synthesis framework, International Journal of Control, Vol. 74, No. 9 , 745-759.

47. Wu F., Yang X., Packard A., and Becker G. (1996), Induced L2 norm control for LPV systems with bounded parameter variation rates, International Journal of Robust and Nonlinear Control, Vol. 6 , No. 9-10, 983-998.
48. Xu F., Tan J., Wang Y., Wang X., Liang B., and Yuan B. (2019), Robust fault detection and set-theoretic UIO for discrete-time LPV systems with state and output equations scheduled by inexact scheduling variables, IEEE Transactions on Automatic Control, Vol. 64, No. 12, 4982-4997.

49. Yang D., Wang Y., and Chen Z. (2020), Robust fault diagnosis and fault tolerant control for PEMFC system based on an augmented LPV observer, International Journal of Hydrogen Energy, Vol. 45, No. 24, 13508-13522.

50. Yeo B.K., and Lu Y. (1999), Array failure correction with a genetic algorithm, IEEE Transactions on Antennas and Propagation, Vol. 47, No. 7, 823-828.

51. Younis W., and Abdelati M. (2009), Design and implementation of an experimental Segway model, AIP Conference Proceedings, Vol. 1107, 350-354.

52. Zhou K., and Doyle J.C. (1998), Essentials of Robust Control, Prentice Hall, Upper Saddle River, NJ. 